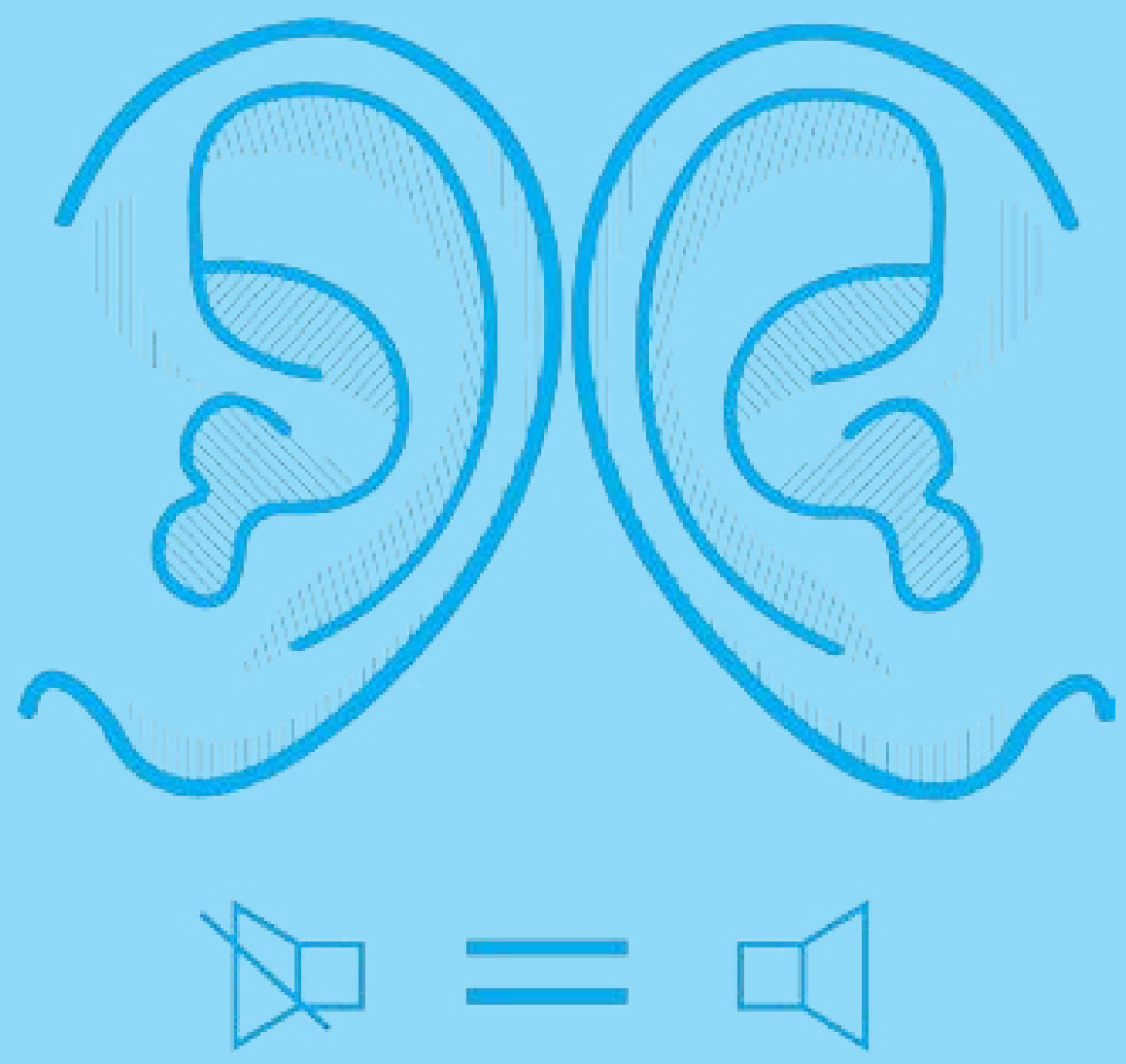

\title{
El aula multidéficit: Una apuesta por la inclusión como alternativa educativa para el proyecto de vida de los estudiantes sordos con discapacidad asociada del Colegio Isabel II. IED
}

The multidéficit classroom: A commitment to inclusion as an educational alternative for the project of life of deaf students with disabilities Associate College Isabel II. IED

A sala de aula multidéficit : Um compromisso com a inclusão como uma alternativa educacional para o projeto de vida dos alunos surdos com deficiência Associate Colégio Isabel II. IED

\section{Camila Andrea Hernández Castillo / Marco Tulio Cárdenas Forero}




\section{Camila Andrea Hernández Castillo ${ }^{1}$ Marco Tulio Cárdenas Forero ${ }^{2}$}

Docente Aula Multidéficit, Colegio Isabel II. Magister en Desarrollo Educativo y Social UPN- CINDE. Correo electrónico: camilaahc@gmail.com

2 Docente Colegio San Francisco de Asís IED. Magister en Desarrollo Educativo y Social UPN-CINDE. Correo electrónico: mtcardenas08@gmail.com

Fecha de recepción: 16 de marzo de 2015 / Fecha de aprobación: 3 de abril de 2015.

\section{Resumen}

El presente artículo muestra una experiencia pedagógica, propuesta en el marco de la inclusión educativa y el enfoque diferencial, denominada "Aula multidéficit", la cual involucra niñas y niños sordos con discapacidades asociadas. Se pretende poner de manifiesto que, más allá de una propuesta para promover procesos de aprendizaje en los estudiantes desde la práctica pedagógica (planteado desde la organización curricular), es una posibilidad para el desarrollo de habilidades para la independencia y la autonomía, en favor de un proyecto de vida.

\section{Palabras clave}

Aula multidéficit, inclusión educativa, habilidades para la independencia y la autonomía, práctica pedagógica.

\section{Summary}

The following article will show a pedagogic experience proposed in the context of inclusive education and the differences called "aula multudeficit" what is involving deaf children with associate disabilities, we are trying to reach further, we want to be more than a proposal to promote learning process to the students with the pedagogic practice (proposed from the curricular organization) it is a chance for the skill's development, the independence and the autonomy in favor to a life project.

\section{Key words:}

Multigrade classroom, inclusive education, skills for life, pedagogical practice.

\section{Resumo}

O presente artigo evidenciara una experiência pedagógica proposta no marco da inclusão educativa e no enfoque diferencial denominado "aula multideficit", que involucra crianças surdas com deficiências associadas. Com isto, pretende-se manifestar que, além de ser uma proposta para promover processos de aprendizado nos alunos desde a prática pedagógica (proposto desde a organização curricular) é uma possibilidade para o desenvolvimento de habilidades para a independência e a autonomia em prol de um projeto de vida.

Palavras chave:

Aula multideficit, inclusão educativa, habilidades para a independência e a autonomía, prática pedagógica. 


\section{A manera de introducción}

$\mathrm{E}$ n la actualidad el paradigma de la educación inclusiva tiene como principio el reconocimiento de la diferencia, tomando como punto de partida las características, particularidades y necesidades de los niños y las niñas para la creación de nuevas estrategias o la flexibilización de las existentes por parte de las instituciones, con el fin de garantizar el acceso, permanencia, cobertura, participación efectiva y el aprendizaje de los estudiantes.

En ese sentido, la presente experiencia busca visibilizar la manera como las practicas pedagógicas desarrolladas al interior de las aulas para sordos se desarrollan en su primera lengua (lengua de señas colombiana), priorizando la creación de estrategias pedagógicas basadas en sus capacidades, competencias, habilidades y potencialidades para la independencia y la autonomía, dejando de lado el discurso de las dificultades, necesidades, carencias, entre otros, conceptos que con el tiempo han guiado los ámbitos en los que hace presencia esta población y que limitaron el acceso y real participación escolar de los mismos.

\section{El contexto: una mirada general}

El Aula Multidéficit ${ }^{3}$ es creada en el año 2011, al interior de la Instrucción Educativa Distrital Isabel II, con el objetivo de brindar una alternativa pedagógica para los estudiantes con diagnóstico de discapacidad cognitiva, neuromuscular, entre otras asociadas a la discapacidad auditiva, cuyos resultados académicos evidenciaban dificultades en el proceso de aprendizaje, alto índice de deserción escolar y una considerable tasa de repetición en los grados cursados.

Por ello, la alternativa "Aula Multidéficit" propone una estrategia de atención pedagógica que permite procesos de formación integral acordes con las características, necesidades, condición de extra-edad, ritmos y procesos de aprendizaje de los niños y

3 La definición de Aula Multidéficit emerge de la propuesta de "Aula Multigrado", expuesta por el equipo de inclusión, del año 2011, a los directivos de la IED Isabel II. Pese a la denominación asignada por las orientaciones pedagógicas de la Secretaría de Educación de Bogotá en su documento "Condiciones de discapacidad y/o excepcionalidad atendidas por la SED", la cual limita el multidéficit únicamente a la sordo-ceguera, el proyecto de aula para sordos se ha denominado como aula multidéficit, pues desde la caracterización de la discapacidad hace referencia a la unión de dos o más discapacidades. niñas allí inscritos. Esto se hace a partir de metodologías y estrategias diversas, fundamentadas en la pedagogía por proyectos de aula, planes de aula, procesos de aprendizaje y desarrollo de habilidades para la independencia y autonomía ${ }^{4}$, fortaleciendo prácticas previas a los procesos de formación pre-vocacional de los niños y niñas.

Hasta 2013 el Aula Multidéficit contaba con dos subgrupos planteados de acuerdo a las habilidades de desarrollo, edad cronológica y tiempo de escolaridad en la institución de los estudiantes. El primero, "Alistamiento pre-vocacional”, estaba conformado por 7 jóvenes de 16 años (edad cronológica), con quienes se realizó, dadas las características enunciadas, un proceso de valoración en una institución especializada en formación vocacional, buscando determinar su estado de preparación para acceder a un contexto diferente al educativo, y realizar la posible inserción al programa de formación para el trabajo y rápida ubicación laboral. El proceso de valoración se desarrolló durante una semana en jornada competa; 3 de los 7 estudiantes valorados cumplieron con los requisitos para ingreso al programa, pero no pudieron acceder por dificultades administrativas.

El segundo grupo, conformado por 6 estudiantes, con un promedio de edad entre los 8 y 15 años, se denominó "Desarrollo de habilidades para la independencia y la autonomía"; por su estructura pedagógica buscó fortalecer las habilidades anteriormente mencionadas, con el objetivo de avanzar hacia otro nivel de nuestra propuesta pedagógica: "Alistamiento pre-vocacional", que en la actualidad (2014) continúa en proceso.

\section{Las prácticas pedagógicas: el for- talecimiento de habilidades para la autonomía y la independencia}

El Aula Multidéficit proyecta en los estudiantes una formación continua como sujetos de derechos, enfocada en el desarrollo de competencias para su autonomía e independencia, basada en prácticas reales a través de diferentes situaciones didácticas que

4 Las habilidades para la independencia y la autonomía son el conjunto de estrategias utilizadas como objeto de aprendizaje desde las prácticas pedagógicas, para ser reorientadas hacia el emprendimiento laboral después de la etapa de escolarización, es decir, son una posibilidad para poder desenvolverse y mantenerse en su etapa adulta y así fortalecer su proyecto de vida. 
propenden por el desarrollo de habilidades y destrezas útiles para la vida fuera de la escuela. En las prácticas pedagógicas se sugiere que las diferencias sirvan como referencia para desarrollar sus actividades y vivir experiencias en el marco del emprendimiento laboral, fortaleciendo su rol como ciudadanos con particularidades y capacidades enriquecidas por valores.

Este escenario no es ajeno a los procesos pedagógicos y curriculares propuestos desde los lineamientos descritos en la Ley General De Educación (115/94), y al Proyecto Educativo Institucional propuesto por el Colegio Isabel II IED, por tal motivo desarrolla sus prácticas pedagógicas, las cuales define como:

[...] el escenario, donde el maestro dispone todos aquellos elementos propios de su personalidad académica y personal. Desde la académica en lo relacionado con su saber disciplinar y didáctico, como también el pedagógico a la hora de reflexionar sobre las fortalezas y debilidades de su quehacer en el aula. En lo personal, el maestro utiliza elementos como el discurso, relaciones intra e inter personales, ya que si no las tiene, es seguro que no obtendrá éxito con la población a la cual va dirigido su conocimiento (Zuluaga, 2013, p. 3).

De este modo, la propuesta pedagógica desarrollada con el grupo en mención, se ha basado en la pedagogía por proyectos de aula (uno por cada período académico), como una: “[...] estrategia metodológica que dinamiza en estudiantes y profesores, la construcción de conocimientos sobre el mundo de la vida de forma contextualizada, experiencial, globalizante y significativa dentro de un ambiente educativo" (INSOR, 2008, p. 13).

Dichos proyectos integran las diferentes áreas del conocimiento bajo una misma problemática a desarrollar, cuyo valor agregado es generar productos (artefactos) a partir de diferentes materiales, con los cuales se aprende sobre el manejo y producción de los mismos, nociones básicas sobre emprendimiento y administración de recursos, entre otros, punto de partida hacia la construcción de su plan de vida.

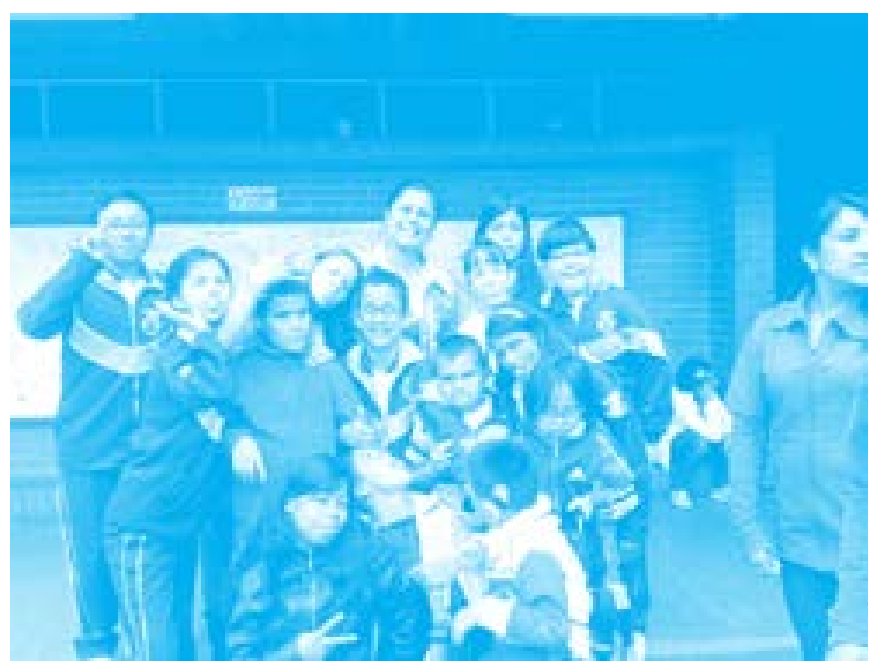

Imagen 1.Maestra, niños y niñas del Aula Multidéficit

\section{Mi planeta: un ejemplo de proyecto pedagógico de aula desde una perspectiva integradora e inclusiva}

\section{El exordio de una práctica pedagógica}

Históricamente la escuela ha sido atravesada por diferentes modelos y enfoques pedagógicos que le han permitido, de una $\mathrm{u}$ otra forma, adquirir un estatus como productora de conocimientos y saberes, de ahí que en la actualidad se den diversas prácticas de orden curricular, e incluso adaptaciones y flexibilizaciones del mismo, las cuales buscan un desarrollo integral de los niños.

De allí que los proyectos pedagógicos de aula emerjan como una posibilidad para el desarrollo y la integración de los estudiantes, ya que su condición activa y metodológica permite explorar e indagar sobre los intereses y necesidades de los niños, del maestro y la escuela, convirtiéndose así en una aproximación a una forma de investigar al interior del aula. Visto de otra manera:

Los proyectos de aula son valiosas oportunidades de enseñanza, aprendizaje y autoformación, que generan actitudes y aptitudes favorables para el trabajo en equipo, la comprensión social y 
práctica del conocimiento científico, la generación de vínculos sociales, la transferencia de conocimiento y el fortalecimiento de la identidad personal, social y profesional (Garzón, 2009, p. 3).

Como se observa, la dinámica propuesta al interior del Aula Multidéficit se enmarca en la implementación de Proyectos Pedagógicos de Aula (PPA), los cuales facilitan la integración de saberes y conocimientos partiendo de los gustos e intereses se los niños. Su implementación se da a partir de prácticas pedagógicas constructivas (Talleres, trabajos independientes, estudio de casos, proyectos, problemas, y laboratorio); socioeconómicas (conversatorios y salidas de campo); lúdicas (dramatizaciones y juegos de roles) y profundizaciones (seminarios).

Además, se integran las áreas del conocimiento relacionándolas con la cotidianidad de cada uno de los niños. Es de resaltar que, desde la perspectiva de Cerda (2001), se inicia el proceso desde las fases del Proyecto Pedagógico, que son: Exploración, donde se hace el diagnóstico general del grupo denotando intereses y gustos; Planeación, en la que se caracterizan y diseñan las prácticas; Ejecución, en la cual se implementan las prácticas y se analiza el impacto, y Evaluación, donde se verifican resultados y se reflexiona sobre las prácticas.

\section{Una mirada holística: la integralidad con las áreas}

El PPA integra las diferentes áreas del conocimiento, de manera que los procesos de enseñanza-aprendizaje articulen las necesidades e intereses de la escuela, de los niños y niñas y de los componentes del currículo, con el horizonte institucional del colegio, generando:

[...] el protagonismo a los alumnos en su proceso de aprendizaje, promoviendo su inventiva, creatividad, curiosidad, el trabajo

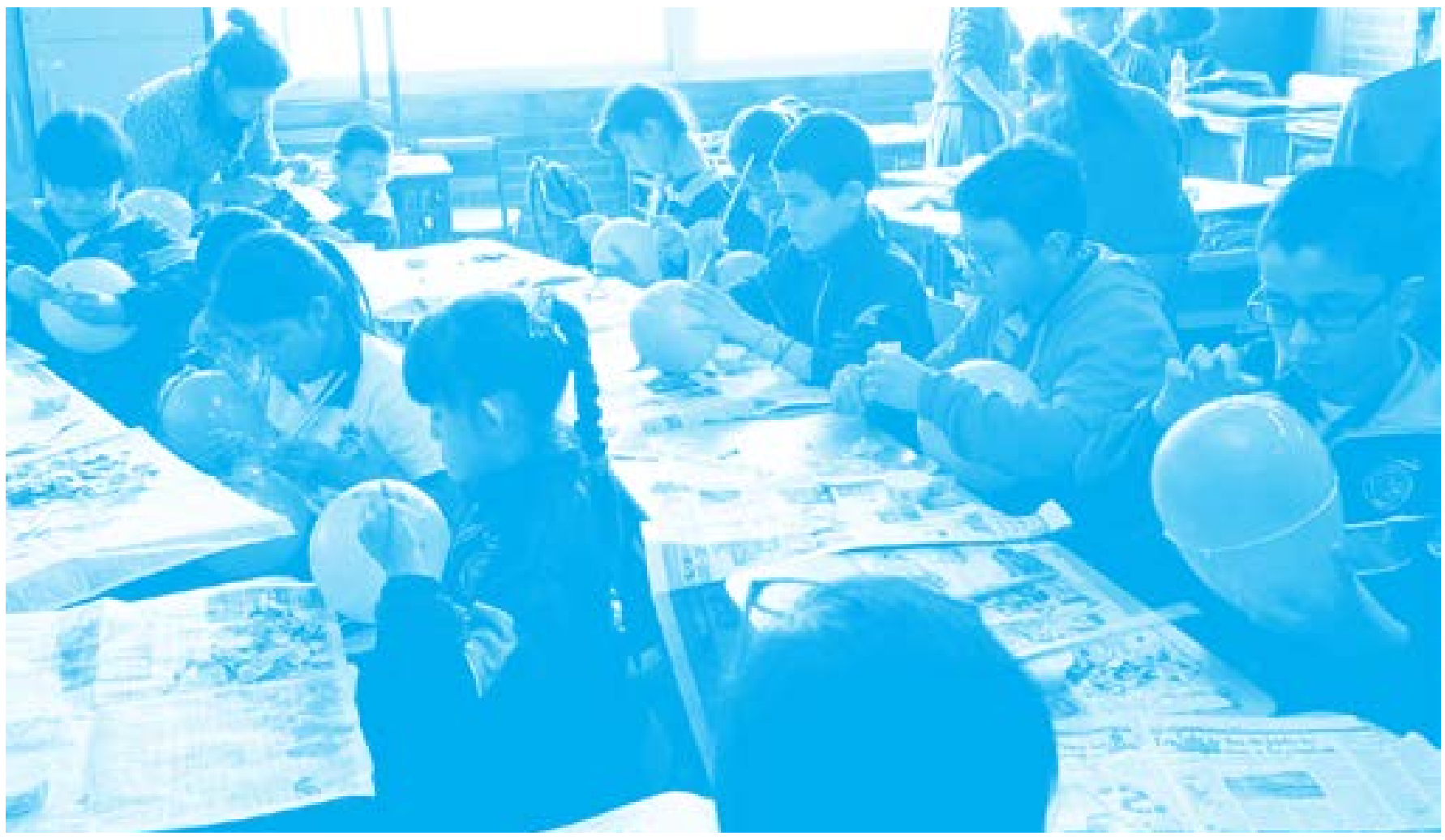

Imagen 2. Practica Pedagógica del PPA: "Mi planeta" 
en equipo, la formulación y la solución de problemas cotidianos y concretos. Los proyectos constituyen una forma de aprender y de enseñar, centrada en los intereses y preocupaciones de los alumnos para que se involucren de forma activa en la planificación, ejecución, presentación y evaluación de experiencias dialogadas de aprendizaje (Guzmán, 2005, p. 9).

A continuación se describe la manera como se articulan las áreas del conocimiento con el PPA.

\section{Las matemáticas y su uso en la cotidianidad}

Teniendo en cuenta los diferentes niveles presentes en el Aula Multidéficit, el PPA “Mi planeta” busca que los niños y niñas se aproximen al concepto lógico-matemático desde su relación con la cotidianidad, es decir:

Hablar sobre las relaciones lógico-matemáticas es referirse a la representación del mundo a través de sistemas y procedimientos por medio de un código propio, integrado por los diversos símbolos matemáticos. [...] es de reconocer que el trabajo se centra en el desarrollo del pensamiento de los niños y las niñas a través de la resolución de problemas de la vida cotidiana, puesto que la matemática básicamente es una práctica social (SED, 2010, p. 187).

Cada una de las etapas de construcción y desarrollo del proyecto permite al estudiante, desde sus competencias, dar soluciones a las preguntas planteadas y asumir roles que le exigen responder frente a cada una de las responsabilidades. Por ejemplo, durante el proceso de producción, deberán mantener cantidades, distribuciones, mediciones, caracterizaciones, conteos y verificaciones, entre otras actividades relacionadas con su cotidianidad. Para reforzar esta práctica, se articularon salidas pedagógicas a escenarios productivos (Central de Abastos CORABASTOS y su entorno comercial), en los cuales se vieron elementos afines a lo aprendido en clase.

\section{Las artes plásticas como herramientas de independencia y autonomía}

Las artes plásticas, dentro del PPA "Mi planeta", permitieron realizar talleres de dibujo, pintura, collage, modelado, construc-

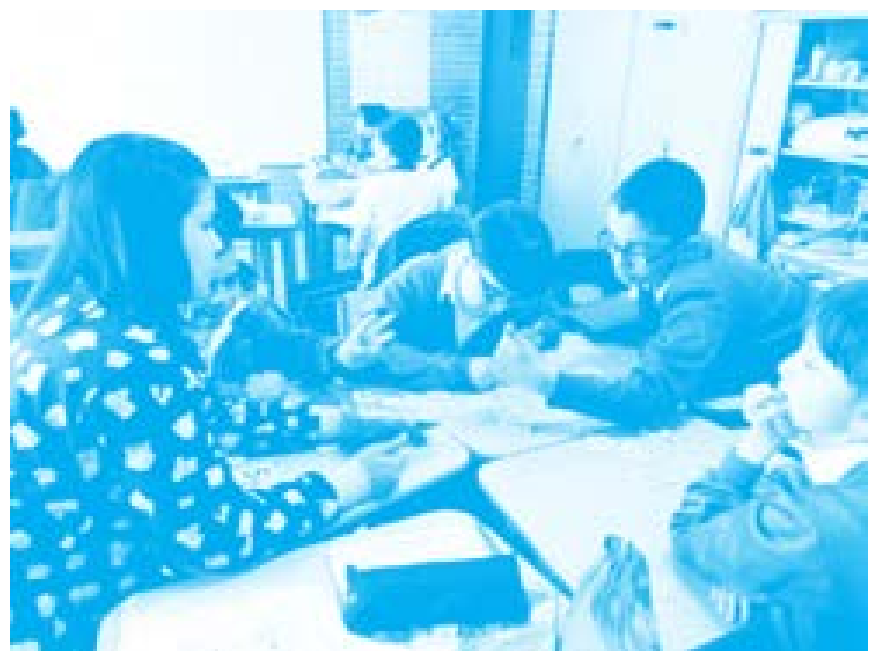

Imagen 3. Clase de matemáticas (Conteo y Seriación)

ciones y escultura, en ellos los niños conocieron, experimentaron y crearon con diferentes materiales, aprovechando las propiedades de los mismos; todo, entendiendo que:

La plástica es motivadora esencial del desarrollo de la percepción, la sensibilidad, la estética, la capacidad creativa y expresiva; no solo permite que los niños y las niñas adquieran un medio para comunicarse y expresarse a través de la imagen, sino que también les posibilita imaginar, inventar e interactuar con los demás, por medio del placer derivado de la experimentación de estímulos visuales y táctiles, en relación con todos los sentidos (SED, 2010, p. 142).

Los diversos proyectos se materializan en la elaboración del producto artístico que los niños comercializan. Con ello, descubren la importancia de reciclar (separar basuras y clasificarlas, entendiendo que el material sea útil para ser reutilizado), reconociendo que dicho proceso es de utilidad para conseguir la materia prima necesaria para el desarrollo de la fase artística del proyecto. Una de las adaptaciones curriculares con las que cuenta la propuesta, ha sido la ampliación de la intensidad horaria para el área artística, lo cual ha favorecido la dedicación y el perfeccionamiento de los productos elaborados. 


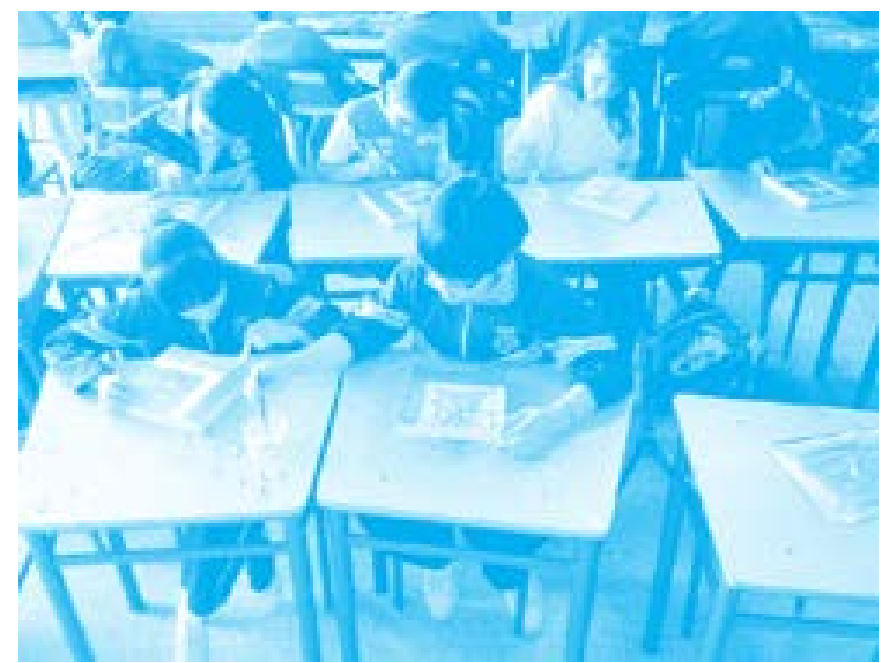

Imagen 4. Clase de artes plásticas. Cuadros navideños para la Feria de Emprendimiento

\section{La lengua de señas como vehículo de cognición}

La práctica comunicativa involucró a los padres de familia y demás agentes del proceso de socialización y adaptación de los niños y niñas, sin dejar de lado la importancia del maestro como mediador de estos procesos; justamente:

El rol del maestro y la maestra cobra vital importancia, reconociendo que las experiencias con el cuerpo, posibilitan el encuentro con el otro desde la empatía, gestándose un constante intercambio de significados a través del lenguaje no verbal que permite, con una gran sensibilidad, comprender esa expresividad corporal y desde allí ofrecer una dinámica de bienestar y estimulación que facilite y acompañe el desarrollo armónico e integral de los niños y niñas (SED, 2010, p.100).

Es de esta forma como se desarrollan las prácticas pedagógicas que contribuyen en la adquisición y fortalecimiento de la lengua de señas, pues es usada en todos los espacios, tanto académicos como sociales, de la institución y el aula. También se han generado espacios de participación con los padres de familia, quienes aprenden diferentes estrategias, basadas en la lengua de señas, para aplicarlas con sus hijos siendo conscientes de la importancia de aprenderla y usarla progresivamente.
Además, durante el desarrollo del proyecto pedagógico, los estudiantes acceden a un espacio mediado por un modelo lingüísti$\operatorname{co}^{5}$ que facilita la creación de situaciones discursivas y potencia los conocimientos que se requieren, las habilidades, destrezas y actitudes que se necesitan para la resolver la problemática planteada en cada período académico. Dicho espacio no solo favorece los conocimientos académicos y contextualizados exigidos por el PPA, sino el reconocimiento de que la persona sorda es sujeto de derechos y deberes.

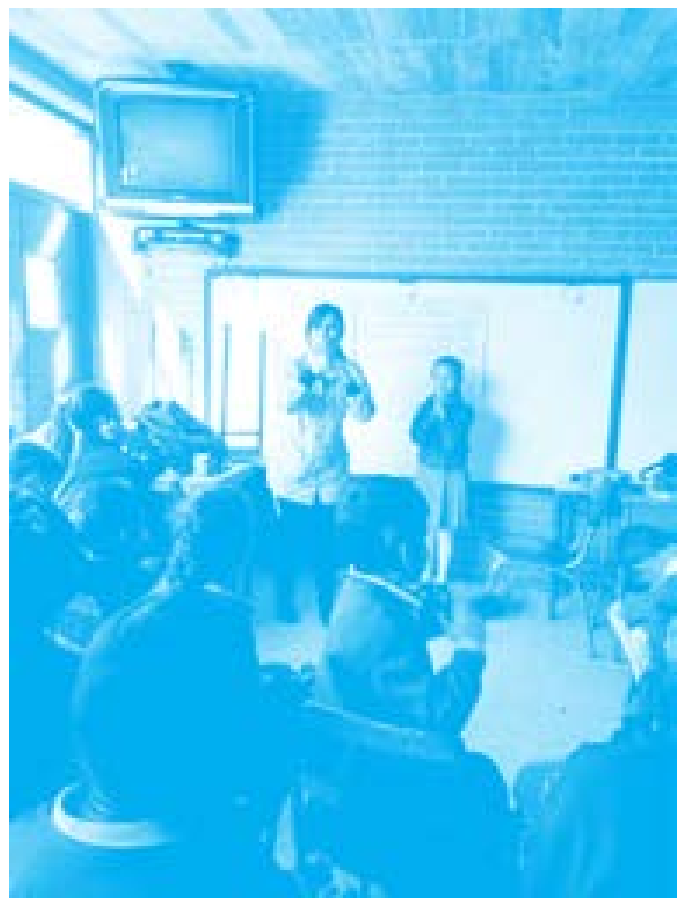

Imagen 5. Clase de Ética en Lengua de Señas

\section{Las ciencias naturales y sociales: una posibilidad para proteger el entorno}

El PPA permite visibilizar diferentes problemas sociales y ambientales a los que los niños y niñas están expuestos, propiciando

5 Con el modelo lingüístico se hace referencia a personas sordas adultas inmersas en el contexto escolar, responsables de agenciar "procesos de adquisición, consolidación y enriquecimiento de la LSC de los estudiantes, y de la enseñanza y cualificación de la misma de los docentes, padres de familia y demás miembros de la comunidad educativa" (SED, 2004, p. 77) 
su participación en la propuesta de soluciones conjuntas o desde el interior del aula, sin embargo es pertinente anotar que:

Más que programar campañas esporádicas de limpieza y reciclaje, el propósito es integrar a las dinámicas diarias de la institución, prácticas ciudadanas que permitan el desarrollo de una conciencia del uso adecuado de los recursos naturales, y que aseguren la apropiación de una cultura en la cual se promueva la valoración por los elementos que conforma el ambiente y el planteamiento de estrategias que busquen mantener un equilibrio entre el medio natural y social, de tal forma que los niños y niñas asuman que el medio ambiente depende de la relación que establecen los grupos humanos con la naturaleza (SED, 2010, p. 182).

Las ciencias sociales y naturales juegan un papel importante en el PPA, pues durante el proceso de identificación de intereses hubo una gran inclinación de los estudiantes por atender los problemas ambientales con los se enfrentan a diario. Por ello, hicieron diferentes propuestas para ayudar al planeta y analizaron las consecuencias de no hacerlo a tiempo; una de las estrategias identificadas fue el reciclaje, por lo que los estudiantes desarrollaron proyectos para separar las basuras y administrar el cuidado del agua, generando campañas publicitarias para cumplir con dicho propósito desde el reconocimiento de los materiales que podían ser reciclados o reutilizados.

\section{La educación física en el ejercicio del reconocimiento de sí y del otro}

Se sugirieron actividades lúdicas y recreativas que involucraran a los niños y niñas en sus diferentes etapas y formas de desarrollo, tales como la participación en salidas pedagógicas (caminatas ecológicas y visitas guiadas al comercio local, destacando la relación con actividades de la cotidianidad) y actividades intra y extramuros, favoreciendo su convivencia y desarrollo integral.

La educación física permite adquirir saberes como la corporalidad (conciencia del cuerpo como agente comunicativo) y la corporeidad (reconocimiento corporal), que contribuyen al reconocimiento de sí mismo como sujeto de derechos, deberes y protector del planeta (responsable de su cuidado). Su ejecución consideró las dificultades de los alumnos a la hora de realizar los ejercicios físicos propuestos en el PPA (de desplazamiento, ejercitación o lúdicos), por lo que las actividades se adaptaron considerando la importancia del reconocimiento del cuerpo (en él se empiezan a establecer características fisicas, biológicas y de género) y la manera como éste hace parte de un espacio y territorio. La apuesta fue articular las practicas pedagogicas a espacios fuera del aula, privilengiando las cualidades fisicas básicas y los patrones básicos de movimiento, ajustados a sus capacidades.

De esta manera, la educación física, y su papel dinamizador del desarrollo corporal, se convirtió en la base para una educación para la vida (habilidades para la autonomía y la independencia), que, más allá de ser la asignatura encargada de la pedagogía del cuerpo, se convirtió en el área que permite la integración y participación de los niños y niñas en sus propios procesos de crecimiento y desarrollo.

\section{La ética como dinámica permanente del grupo}

La constante reflexión acerca de los valores implícitos en cada una de las prácticas pedagógicas, guarda una estrecha relación con la manera como socializan los niños y niñas del aula, y con el conocimiento de los espacios en los que interactúan con los demás. Es por eso que se hace necesario involucrar los deberes y derechos, como niños y estudiantes, en el ejercicio para la convivencia pacífica. Todo esto a favor de reafirmarse como:

\footnotetext{
$[\ldots]$ seres sociales y a la vez únicos y particulares que hacen parte activa de la vida cultural en la que crecen y viven, en donde se compenetra la construcción de autonomía e identidad con los otros en la convivencia. De ahí la importancia de generar espacios y estrategias en las que los niños y las niñas se vean en la necesidad de interactuar con los otros en medio de charlas de grupo, juegos de equipo y preguntas en las que confluyan intereses comunes, de ellos y ellas; de manera que inicien el reconocimiento del otro y la afirmación de sí mismos en el desarrollo de diversas experiencias (SED, 2010, p. 93).
}

Dentro del PPA, se emplea el Aprendizaje Cooperativo como metodología de trabajo con los niños y niñas, convirtiéndose en el eje transversal de todas las áreas, pues es desde allí que todos los estudiantes participan de manera igualitaria en el desarrollo de las diferentes etapas propuestas; todos se involucran según sus desempeños, intereses y asignación de tareas y roles. 


\section{La evaluación como estrategia para el mejoramiento}

El Aula Multidéficit atiende estudiantes con una serie de necesidades y dificultades comunes, individuales o especiales, de ahí que en un primer momento la evaluación, (diagnóstica) permite dar cuenta del estado de cada niño y niña para, posteriormente, flexibilizar las prácticas pedagógicas y evaluativas, acomodándolas a cada particularidad.

La evaluación invita al reconocimiento de la diversidad, donde es pertinente reconocer que los estudiantes tienen condiciones singulares y propias que los particularizan, precisamente porque tienen una historia que es del orden de lo personal y que cuando hay alteraciones o dificultades en su desempeño, valga decir en el aprendizaje, es necesario dirigir la mirada hacia las condiciones que afectaron sus dimensiones del desarrollo personal y los entornos escolares y familiares (Villareal, 2004, p. 10).

Luego del diagnóstico, la evaluación se convierte en "evaluación formativa", ya que se utiliza como estrategia permanente para mejorar algunos procesos de aprendizaje (los que posiblemente pueden ser profundizados); en este momento se destaca la co-evaluación como actividad constante, pues permite avanzar en acciones para conseguir las metas u objetivos previstos dentro del PPA. No se puede dejar de lado que este estado de la evaluación va de la mano con la estimulación emocional, que fortalece sus vínculos sociales.

Sin embargo, los alcances de los niños y niñas deben ser cuantificados dentro del Sistema Institucional de Evaluación, situación que hace evidente que, en la actualidad, evaluar desde la inclusión es una actividad compleja cuando se hace parte de un sistema educativo, ya que, según las formas de evaluar propuestas desde el currículo, siempre hay una tendencia a homogenizar a los estudiantes.

\section{A modo de cierre}

Como se observó, la estrategia "Aula Multidéficit", fundamentada en Proyectos Pedagógicos de Aula, es una alternativa que permite favorecer el desarrollo de habilidades y destrezas para la independencia y la autonomía de los niños y niñas; por lo que se propone implementarla en las aulas regulares, para que sea posible propiciar en los estudiantes, desde el comienzo, el desarrollo de competencias en la vida. Este tipo de experiencias permite destacar al saber como pieza clave en la formación de ciudadanos y sujetos productivos para la sociedad, sin importar su condición de diferencia. 


\section{Referencias}

Cerda, H. (2001). La pedagogía de proyectos: algo más que una estrategia. Bogotá: Magisterio.

Garzón, E. Y. (2009). Guía para la estructuración de Proyectos de Aula. Tunja: Universidad de Boyacá.

Guzmán, A. (2005). Los proyectos pedagógicos de aula. Caracas: Universidad Rómulo Gallegos.

INSOR. (2008). Los proyectos pedagógicos y la lengua escrita en la educacion bilingüe y bicultural para sordos. Bogotá: Ministerio de Educación Nacional.

SED. (2004). Integracion escolar en sordos, usuarios de lengua de señas colombiana en colegios distritales. Bogotá: SED.
SED. (2010). Experiencia plástica. Lineamiento pedagógico y curricular para la educación inicial en el Distrito. Bogotá: SED.

Villareal, J. E. (2004). Evaluación pedagógica para la inclusión educativa. Medellin: Universidad de Antioquia.

Zuluaga, O. L. (2013-Abril 4). Pedagogía: un concepto de práctica pedagógico. Obtenido el 15 de Enero de 2015, desde http://bibliotecadejuan.blogspot.com/.../pedagogia-un-concepto-de-práctica 